\title{
Alleviation of insulin resistance and liver damage by oral administration of Imm I 24-E is mediated by increased Tregs and associated with increased serum GLP-I and adiponectin: results of a phase I/II clinical trial in NASH
}

This article was published in the following Dove Press journal:

Journal of Inflammation Research

19 December 2012

Number of times this article has been viewed

\section{Meir Mizrahi' \\ Yehudit Shabat' \\ Ami Ben Ya'acov' \\ Gadi Lalazar' \\ Tomer Adar' \\ Victor Wong ${ }^{2}$ \\ Brian Muller ${ }^{2}$ \\ Grant Rawlin² \\ Yaron Ilan'}

'Liver Unit, Hebrew UniversityHadassah Medical Center, Jerusalem, Israel; ${ }^{2}$ mmuron Limited, North Melbourne, Australia
Correspondence: Ami Ben Ya'acov Liver Unit, Hebrew University-Hadassah Medical Center, Ein-Karem, Jerusalem, Israel, POB 1200

Tel +972 26777559

Email amib@hadassah.org.il
Background: Nonalcoholic steatohepatitis (NASH) is considered to be part of the nonalcoholic fatty liver disorders and its incidence is increasing. Imm124-E (Immuron Ltd, Melbourne, Australia), containing hyperimmune bovine colostrum, has been shown to exert an immunomodulatory effect and to alleviate target organ damage in animal models of NASH. The aim of our study was to determine the safety and efficacy of oral administration of Imm124-E to patients with insulin resistance and NASH.

Methods: In an open-label trial, ten patients with biopsy-proven NASH and insulin resistance were orally treated with Imm124-E for 30 days.

Results: Oral administration of Imm124-E was safe, and no side effects were noted. Alleviation of insulin resistance was reflected by significantly improved hemoglobin $A_{1 c}\left(\mathrm{HbA}_{1 c}\right)$ values in all ten treated patients. For between five and eight responders, the following effects were noted: a decrease in fasting glucose levels; improved oral glucose tolerance test (OGGT) and homeostatic model assessment insulin resistance (HOMA) scores; and alleviation in lipid profile. These effects were accompanied by increased serum levels of glucagon-like peptide 1 (GLP-1), adiponectin and T regulatory cells.

Conclusion: Hyperimmune colostrum alleviates NASH.

Keywords: NASH, anti-LPS, diabetes, adipokines, regulatory $\mathrm{T}$ cells

\section{Introduction}

Nonalcoholic fatty liver disease (NAFLD) is the most common of all liver disorders. ${ }^{1-5}$ The spectrum of histological abnormalities defined by NAFLD includes simple steatosis and nonalcoholic steatohepatitis (NASH), as its more extreme form. ${ }^{6}$ NASH has emerged as the major cause of chronic liver disease in developed countries. ${ }^{7}$ The high prevalence of these conditions is expected to increase concurrent with the epidemics of obesity and type 2 diabetes mellitus, which are the major risk factors for NAFLD and NASH. ${ }^{8}$ The pathogenesis of NASH is unknown; however, the hypothesis that NASH is associated with peripheral insulin resistance has been intensively explored. ${ }^{9}$

Indeed, patients with NASH and fatty liver were found to have higher serum insulin levels than normal individuals..$^{9,10}$ No therapy has been proven effective for patients with NASH. 
Bovine colostrum (BC) is the milk of lactating mammals that is secreted during the first 72 hours following birth. BC differs from regular milk and contains abundant bioactive components - including growth factors, immunoglobulins, lactoperoxidase, lysozyme, lactoferrin, nucleosides, vitamins, peptides, and oligosaccharides - that are of increasing relevance to human health. ${ }^{11,12}$ The value of $\mathrm{BC}$ as a biological medicine was well documented in several clinical trials. ${ }^{13}$ Colostrum has been shown to balance blood sugar levels, and a suggested mechanism for this is associated with its high content of insulin-like growth factor (IGF-1), which can stimulate glucose utilization and may be beneficial for the treatment of diabetic patients. But the main actions of $\mathrm{BC}$ include antibacterial effects and a modulation of the immune response. The ability of BC to neutralize lipopolysaccharides (LPS) and to inhibit enteropathogenic endotoxemia in animal models was investigated. ${ }^{13}$ Oral administration of $\mathrm{BC}$ was shown to reduce the influx of LPS from the gut and this appears to be a major mechanism underlying BC's therapeutic effect. ${ }^{13}$

Immunoglobulins are the main immune components of the acquired immune system presented in colostrum. IgG is the major immunoglobulin class present in ruminant milk, in contrast to IgA being the major immunoglobulin present in human milk. ${ }^{13,14}$ The immunological activity of bovine $\operatorname{IgG}$ in milk from cows immunized against human pathogens is similar to that of IgG in human milk, demonstrating the benefit of hyperimmune bovine milk in the human diet. ${ }^{15,16}$ Skim milk from cows immunized with human enteropathogenic microorganisms provides protection on cholesterol metabolism that has been demonstrated in rodents and in clinical trials. ${ }^{17-19}$

Regulatory T cells (Tregs) are essential in controlling a variety of immune responses. These responses involve some inflammation pathways that are well accepted as key elements in obesity-associated insulin resistance. Recently, the impact of Tregs on the glucose homeostasis in mice with diet-induced obesity was explored. CD4+ T lymphocytes resident in visceral adipose tissue were shown to control insulin resistance in mice with diet-induced obesity, and analyses of human tissue suggested that a similar process may also occur in humans. ${ }^{20}$ Another study reported that CD4+ Foxp3+ cells with a unique phenotype were highly enriched in the abdominal fat of normal mice, but their numbers were strikingly reduced in insulin-resistant models of obesity. ${ }^{21}$

A study that was conducted in our laboratory showed that induction of NKT cells by hyperimmune BC (enriched with anti-LPS antibodies) had an ameliorating effect in ob/ ob mice. ${ }^{22}$
The aim of the present pilot study was to determine the safety of oral administration of Imm124-E, an anti-LPS hyperimmune $\mathrm{BC}$, in humans; and to assess its immunomodulatory effects on Tregs, glucose homeostasis, various serum cytokines, and lipid profile, in NASH patients.

Although the medical evidence of a benefit in this small study is limited, our data show that Imm124-E is safe and was able to improve glycemic control. This beneficial effect was accompanied by improvements of insulin resistance and hyperlipidemia, and changes in the level of cytokines and several subsets of Tregs.

These data suggest a commercially useful procedure for preparing hyperimmune $\mathrm{BC}$ containing high levels of anti-LPS antibodies, that could allow production of a safe treatment for NASH.

\section{Methods}

\section{Patient population}

This was an open-label and single-center trial that was conducted in patients with type 2 diabetes and NASH. The study was performed in accordance with the guidelines of the Hebrew University-Hadassah Institutional Committee for Human Clinical Trials and with the approval of the Israel Ministry of Health Committee for Human Trials. All patients provided written informed consent before the study.

\section{Inclusion criteria}

Ten patients were enrolled in the open-label trial. Participants (men and women between 18 and 60 years old) were evaluated for eligibility after they signed a written informed-consent form. The diagnosis of NASH was based on a liver biopsy score of 4 or above and altered glucose metabolism, including diabetes (nontreated or treated with up to two drugs without any change in medication during the two months prior to enrollment), impaired fasting glucose or impaired glucose tolerance, and hemoglobin $\mathrm{A}_{1 \mathrm{c}}\left(\mathrm{HbA}_{1 \mathrm{c}}\right)$ between $5.5 \%$ and $14 \%$. Impaired fasting glucose was defined as $>100 \mathrm{mg} / \mathrm{dL}$. Impaired glucose tolerance was defined as a blood sugar level $>140 \mathrm{mg} / \mathrm{dL} 2$ hours post-glucose load and an $\mathrm{HbA}_{1 \mathrm{c}}$ between $5.5 \%$ and $8 \%$. No evidence of other viral or immunemediated liver disease was present.

\section{Exclusion criteria}

Patients meeting any of the following criteria were excluded: active coinfection with hepatitis $\mathrm{A}, \mathrm{B}$, or $\mathrm{C}$ viruses; the presence of human immunodeficiency virus (HIV) infection, hepatocellular carcinoma, fulminant liver failure, severe deteriorating synthetic liver functions, or a clinically significant 
infectious, immune-mediated or malignant disease; any history of treatment with immunomodulatory drugs, including steroids and nonsteroidal anti-inflammatory drugs (NSAID), at any time within the previous four weeks; a history of coagulopathy; women with childbearing potential unless surgically sterile or using adequate contraception (ie, IUD, oral or Depo-Provera contraceptive or barrier plus spermicidal); anemia $(\mathrm{Hb}<10.5 \mathrm{gm} / \mathrm{dL})$, thrombocytopenia (platelets $<100 \mathrm{~K} / \mu \mathrm{L}$ ) or lymphopenia (absolute lymphocyte count $<0.7 \times 10_{9} / \mathrm{L}$ ); or allergy to cow milk or lactose intolerant.

\section{Therapy and laboratory follow up}

After complete medical evaluation and liver biopsy, patients who qualified for therapy were given Imm124-E (Immuron Ltd, Melbourne, Australia), an anti-LPS hyperimmune BC, in a dose of $600 \mathrm{mg}$ three times daily (total of $1800 \mathrm{mg}$ /day) for 30 days. Patients were followed for 60 days through regular weekly scheduled visits that included physical examination, ongoing medical history review, and laboratory tests. Safety was assessed by monitoring the patients for adverse events. Blood was drawn at each visit for determination of complete blood counts (CBC), sedimentation rate (ESR), and standard chemistries, including liver enzymes, international normalized ratio (INR), lipid profile, C-reactive protein (CRP), $\mathrm{HbA}_{1 \mathrm{c}}$, and serum insulin level. All patients underwent a repeat oral glucose tolerance test (OGTT) and a homeostatic model of assessment insulin resistance (HOMA) score evaluation at the end of the study.

\section{Primary endpoint}

The safety of oral administration of Imm124-E was the primary endpoint.

\section{Secondary endpoints}

The secondary endpoints included reduction of the liver enzymes alanine aminotransferase (ALT), aspartate aminotransferase (AST) and gamma-glutamyl transpeptidase $(\gamma-\mathrm{GT})$ by $10 \%$, improvement in insulin sensitivity (Matsuda index from oral glucose tolerance assessment), and improved lipid profile.

The levels of liver enzymes (AST and ALT, alkaline phosphatase [AP] and $\gamma$-GT), serum fasting glucose, insulin, and plasma lipids (cholesterol and triglycerides) were determined using standard methods.

\section{Colostrum collection, processing and preparation}

Imm124-E was prepared from cows immunized with a vaccine containing an extract of the surface antigens from the most common varieties of enterotoxigenic $E$. coli. To prepare each batch of Imm124-E powder, colostrum was collected appropriately from immunized cows and was frozen in individual bags for testing. For processing, colostrum was thawed and pooled, and the fat was removed. Each batch was pasteurized. Colostrum was then concentrated by ultrafiltration to reduce the volume before freeze-drying. The ultrafiltration step reduced lactose in the final powder to less than $7 \%$, compared with about $50 \%$ in skim milk powder. Colostrum was manufactured and then tested by an accredited testing lab (Dairy Technical Services, Kensingtone, VIC, Australia) against specifications for protein, moisture, lactose, fat, antibiotics, and microbiology parameters.

\section{Flow cytometry}

Peripheral blood mononuclear cells (PBMCs) from blood samples collected at day 0 and day 30 were isolated using a Ficoll-Hypaque gradient. Cells were resuspended in Phosphate buffered saline (PBS) containing 1\% bovine serum albumin (BSA). For surface staining, PBMCs were incubated with either fluorochrome-conjugated antibodies against the indicated cell surface markers at the recommended dilution or with isotype control antibodies, for 30 minutes at $4^{\circ} \mathrm{C}$. We used the following cell surface antibodies: CD4-FITC, CD25-PE, CD8-FITC, CD56-FITC, CD69-PE, CD3-APC, CD62-PE, and HLA-DR-APC (eBioscience Inc, San Diego, CA). Cells were then washed in PBS containing 1\% BSA and fixed with fixation buffer (eBiosciences) for another 50 minutes. For intracellular staining of Foxp3, cells were permeabilized with Foxp3 staining buffer (eBioscience) after fixation and stained with APC-conjugated antibodies against Foxp3 (eBioscience). The stained cells were then washed twice and resuspended in $250 \mu \mathrm{L}$ of PBS containing $1 \% \mathrm{BSA}$ and kept at $4^{\circ} \mathrm{C}$. A total of $10^{6}$ stained cells in $250 \mu \mathrm{L}$ of PBS containing 1\% BSA were subsequently analyzed using a FACS LSR II instrument (BD Biosciences, Franklin Lakes, NJ) with the FCS express V.3 software (DeNovo Software, Los Angeles, CA). Only live cells were counted, and the background fluorescence from non-antibody-treated lymphocytes was subtracted.

\section{Measurements of circulating cytokines and adiponectin}

Blood was drawn from all patients on day 1 and on day 30 of the study. Serum levels of interleukin-6 (IL-6) were determined using a "sandwich" enzyme-linked immunosorbent assay (ELISA) using commercial kits (Quantikine ${ }^{\circledR} ;$ R\&D Systems Inc, Minneapolis, MN), according to the manufacturer's 
instructions. Glucagon-like peptide-1 (GLP-1) was tested by the following method: Blood was collected from all patients after a 12-hour overnight fasting at 180 minutes time point of OGTT. The blood was collected in ice-cooled EDTA tubes and immediately (<30 seconds) after collection, $20 \mu \mathrm{L}$ of dipeptidyl peptidase 4 (DPP-4) inhibitor was added to $2 \mathrm{~mL}$ of plasma, according to manufacturer's instructions. The tubes were inverted and were then centrifuged immediately at $1000 \times \mathrm{g}$ for 10 minutes in a refrigerated centrifuge. After plasma separation, the specimens were stored at $-70^{\circ} \mathrm{C}$ until the ELISA assay was run. The circulating level of human GLP-1 was quantified using a commercial ELISA kit from Millipore Corp (Billerica, MA) according to the manufacturer's instructions. Serum levels of human adiponectin were determined using a commercial ELISA kit from Linco Research Inc (St Charles, MO). Serum was diluted 500 fold before the assay, and $20 \mu \mathrm{L}$ of diluted serum, standard samples and controls, were plated in duplicate on a mouse anti-human adiponectin-coated plate and examined using an ELISA reader at $450 \mathrm{~nm}$, according to the manufacturer's instructions.

\section{HOMA score}

HOMA is designed to predict the homeostatic concentrations of fasting insulin and glucose, which arise from varying degrees of beta-cell deficiency and insulin resistance. The model is nonlinear, but can be simply approximated. Two types of HOMA scores are currently being evaluated in clinical practice for determining fasting glucose and insulin levels. HOMA IR $=$ insulin resistance $=$ (fasting insulin in $\mathrm{mU} / \mathrm{L}) \times($ fasting plasma glucose in $\mathrm{mmol} / \mathrm{L}) / 22.5$. HOMA B = beta-cell function [\%] $=20 \times($ fasting insulin in $\mathrm{mU} / \mathrm{L}) /([$ fasting glucose in $\mathrm{mmol} / \mathrm{L}]-3.5)$.

In our study, we have used the HOMA IR levels.

\section{Sample size and power}

This study was not geared to detect rarely occurring treatment-associated adverse events. Summary statistics of all clinical and laboratory variables were calculated by time point; Paired $t$-tests were performed to test the statistical significance of all the before and after treatment measures. A two-tailed $P$-value of 0.05 was considered statistically significant.

\section{Results}

\section{Determination of anti-LPS antibodies in Imm I 24-E}

Details about the IgG antibodies in Imm124-E (an anti-LPS hyperimmune BC) obtained post-cow's immunization with several strains of enteropathogenic $E$. coli were recently published. $^{22}$ The specific antibody titers in Imm124-E were analyzed by a validated in-house ELISA against a pool of antigens from these strains of E. coli. ${ }^{22}$

\section{Safety parameters}

All patients completed the study according to the protocol. No treatment-related adverse events were noted in any of the clinical and laboratory parameters tested during treatment or follow up.

\section{Imm I 24-E ameliorates insulin resistance}

To determine the effect of Imm124-E on the glycemic control in NASH patients, especially in the diabetic patients with impaired glucose tolerance, we tested several parameters. A recommended target of $\mathrm{HbA}_{1 \mathrm{c}}$ in clinical trials is a $6.5 \%$ to $7.0 \%$ decrease. ${ }^{23}$ Figure $1 \mathrm{~A}$ shows the significant improvement in $\mathrm{HbA}_{1 \mathrm{c}}$ values in all ten treated patients $(7.49 \%$ vs $6.38 \%$ for day 1 vs day 30 , respectively). The treatment with Imm124-E for 30 days caused a $14.8 \%$ decrease in $\mathrm{HbA}_{1 \mathrm{c}}$ values in all ten patients. Figure 1B shows that Imm124-E also exerted a beneficial effect on insulin resistance, as assessed by measuring the early peak of insulin secretion at 30 minutes after glucose administration (OGTT of $75 \mathrm{~g}$ of glucose); after 30 minutes, insulin secretion increased from 310 to $538.4 \mathrm{pmol} / \mathrm{L}$ for day 1 vs day 30 , in five of the treated patients.

Figure $1 \mathrm{C}$ shows a reduction in the fasting plasma glucose levels of treated patients (6.3 vs $5.8 \mathrm{mmol} / \mathrm{L}$ for day $1 \mathrm{vs}$ day 30 , for five patients). Figure $1 \mathrm{D}$ shows an improvement $(P=0.08)$ in the results of the OGTT, as indicated by the area under the curve (2492 vs 2252 for day 1 vs day 30, respectively, for five patients), and Figure 1E shows the improved HOMA scores (6.7 vs 4.8 for day 1 vs day 30, respectively, for six patients).

Taken together, these results show an improvement in insulin resistance in patients treated with Imm124-E.

\section{Oral administration of Imm I 24-E increases regulatory $T$ cells}

In obesity-related disorders, such as NASH, chronic local inflammation is present in adipose tissue, and cells of the innate immune system are crucially involved in adipose inflammation and systemic metabolic abnormalities. ${ }^{24}$ We characterized several subsets of peripheral $\mathrm{T}$ cells from PBMCs using flow cytometry. Figure 2A shows that CD4+CD25+ cells were elevated 30 days after oral treatment with Imm124-E in 7 out of the 10 patients (4.63\% vs $6.28 \%$ for 
A

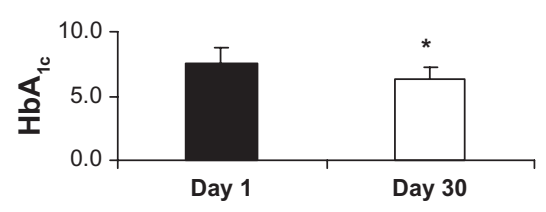

C

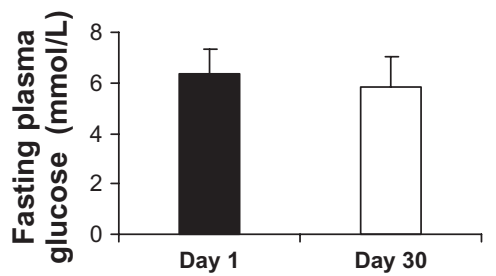

B

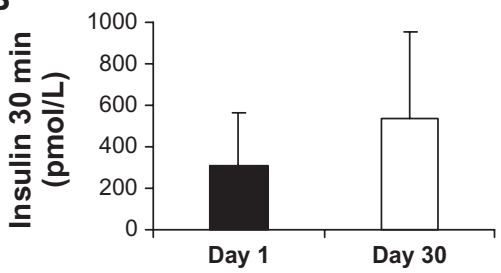

D

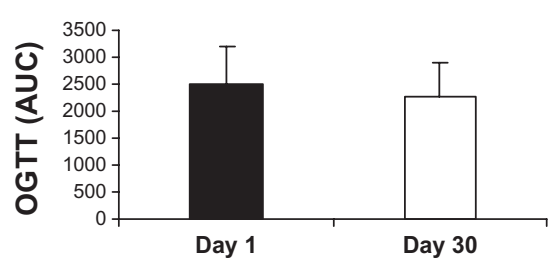

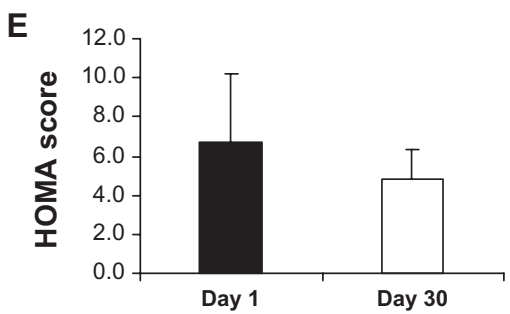

Figure I Effect of Imm I24-E on insulin resistance, as measured on days I and 30. Graphs show (A) The mean percentage of HbA in all ten treated patients; (B) Serum insulin 30 minutes after starting the OGTT, in five treated patients. The serum insulin level was measured after overnight fasting and oral administration of $75 \mathrm{~g}$ of glucose; (C) Fasting plasma glucose levels in five treated patients. The fasting plasma glucose levels were measured after overnight fasting; (D) The mean percentage of OGTT in five treated patients. The assay was performed every 30 minutes for 3 hours; (E) HOMA score calculation was applied as $\mathrm{HOMA} I R=($ fasting insulin in $\mathrm{mU} / \mathrm{L}) \times($ fasting plasma glucose in $\mathrm{mmol} / \mathrm{L}) / 22.5$. Graphs $(\mathbf{A}-\mathbf{C})$ indicate means $\pm \mathrm{SD}$; Graph (D) indicates means $\pm \mathrm{SD}$ of AUC.

Note: $* P<0.03, t$-test.

Abbreviations: $\mathrm{HbA}_{\mathrm{lc}}$, hemoglobin $\mathrm{A}_{\mathrm{lc}}$; OGTT, oral glucose tolerance test; AUC, area under the curve; HOMA, homeostasis model assessment; HOMA IR, insulin resistance; SD, standard deviation.

day 1 vs day 30, respectively). This elevation is demonstrated in Figure 2B with representative fluorescence-activated cell sorting (FACS) plots taken from the analysis of two patients. A significant $(P=0.002)$ increase in CD4+CD25+ HLA-DR cells ( $2.3 \%$ vs $3.8 \%$, gated on CD4, for day 1 vs day 30$)$ was noted in the PBMCs of seven treated patients, as presented in Figure 2C. A representative dot plot of CD4+CD25+ HLA-DR cells is shown in Figure 2D. An increase in CD4+CD25+Foxp3+ cells was noted in seven out of ten treated patients (Figure 2E, $1.7 \%$ vs $2.2 \%$, in responders). Figure $2 \mathrm{~F}$ shows an increase in $\mathrm{CD} 4+\mathrm{CD} 62+$ cells that was noted in six patients $(36.5 \%$ vs $41 \%$, in responders, gated for CD4 cells). Taken together, the data presented here show that oral administration of Imm124-E was associated with alterations of regulatory $\mathrm{T}$ lymphocytes, which may contribute to some of its anti-inflammatory effects.

\section{Effect of Imm I 24-E on serum levels IL-6}

Figure 3A shows the net increase in serum IL-6 levels in six treated patients ( $4.6 \mathrm{vs} 5.5 \mathrm{pg} / \mathrm{mL}$, in responders) in response to oral administration of Imm124-E, as measured by ELISA.

\section{Effect of oral administration of Imm I 24-E on serum levels of GLP-I and adiponectin}

The central role of GLP-1 in glucose tolerance has raised questions about the possible involvement of this peptide in the pathogenesis of diabetes. A recent study found that the sensitivity of diabetic patients to GLP-1 was significantly reduced relative to nondiabetic individuals. ${ }^{25}$ We compared the circulating levels of serum GLP-1 before and after carrying out OGTT on day 1 and on day 30. Figure 3B shows that oral treatment with Imm124-E for 30 days increased serum levels of GLP-1 post-OGTT in five of the treated patients (6.31 vs $6.78 \times 10^{4} \mathrm{pM}$, in responders). Figure $3 \mathrm{C}$ shows the serum levels of adiponectin, which were increased in eight patients (6181 vs $7069 \mathrm{ng} / \mathrm{mL}$ ).

\section{Effect of Imm I24-E on lipid profile}

Figure 4A shows a beneficial effect of the Imm124-E on serum levels of total cholesterol in five treated patients (5.8 vs $4.5 \mu \mathrm{mol} / \mathrm{L}$, for day 1 vs day 30 , respectively). Similar results were obtained with serum low-density lipoproteins (LDL) measurements (3.8 vs $2.7 \mu \mathrm{mol} / \mathrm{L}$, for 
A

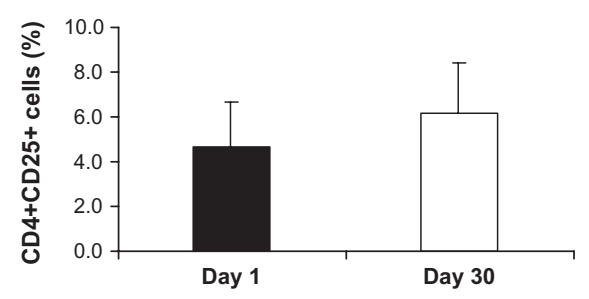

C

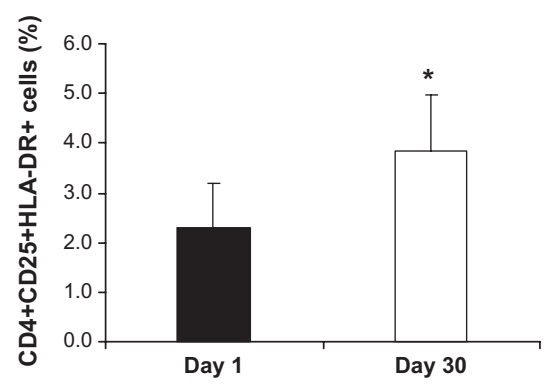

E

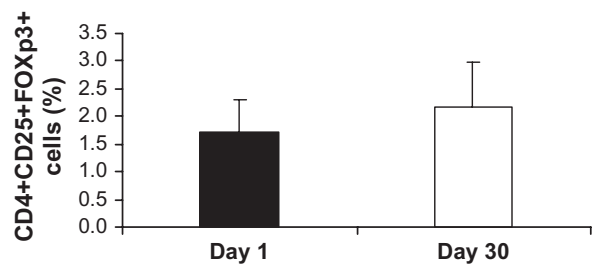

B

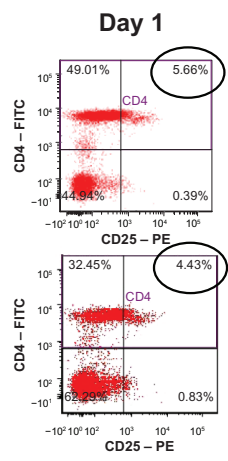

D
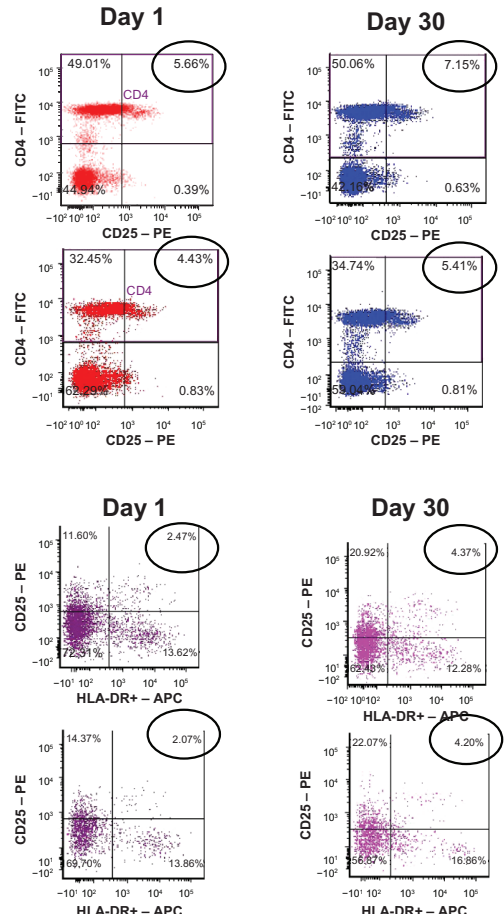

Day 30

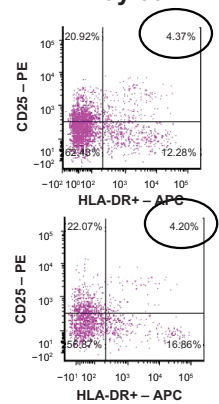

F

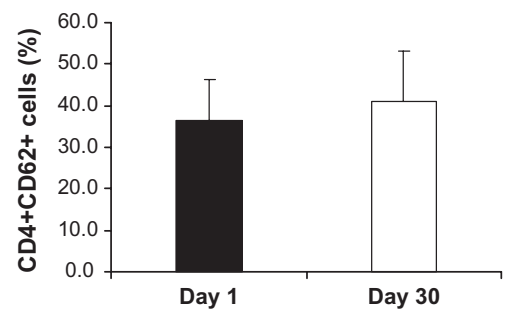

Figure 2 Effect of Imm I24-E on Tregs in PBMCs. PBMCs were isolated from patients treated with Imm I24-E at day I and at day 30. The cells were stained for various cell markers and then analyzed using flow cytometry. Graphs show (A) The mean percentage of CD4+CD25+ cells from seven patients; (B) Representative FACS plots taken from two patients showing the increase in the distribution of CD4+CD25+ cells (circled). The numbers in the top right quadrate indicate the frequency of CD4+CD25+ cells, gated on CD4; (C) The mean percentage of CD4+CD25+HLA-DR+ cells from seven patients; (D) Representative FACS plots taken from two patients showing the increase in CD4+CD25+HLA-DR+ cells (circled). The numbers in the top right quadrate indicate the frequency of CD4+CD25+HLA-DR+ cells, gated on CD4; (E) The mean percentage of CD4+CD25+FOXP3+ cells from five patients; (F) The mean percentage of CD4+CD62L+ cells from six patients. Graphs (A, C, E and $\mathbf{F})$ indicate means \pm SD.

Note: $* P=0.002$, $t$-test.

Abbreviations: Tregs, regulatory T cells; PBMC, peripheral blood mononuclear cells; FACS, fluorescence-activated cell sorting; SD, standard deviation.

day 1 vs day 30, respectively, Figure 4B). A slight improvement in serum triglycerides levels was noted in five patients (data not shown).

The data presented here suggest that oral administration of Imm124-E has an ameliorating effect on the lipid profile in treated NASH patients.

\section{Effect of oral administration of Imm I 24-E on liver injury}

Figure 5A-D show the effect of oral administration of Imm124-E on serum levels of ALT, AST, AP and $\gamma$-GT, respectively. ALT levels were decreased in five patients (57.4 vs $48.6 \mu / \mathrm{L}$, for day 1 vs day 30$)$. AST levels were decreased in five of the treated subjects $(51.2$ vs $44.6 \mu / \mathrm{L}$, for day 1 vs day 30). AP levels were improved in eight of the treated patients (83.1 vs $73.9 \mu / \mathrm{L}$, for day 1 vs day 30$)$, and $\gamma$-GT levels were decreased in five treated patients ( $88.2 \mathrm{vs}$ $73.2 \mu / \mathrm{L}$, for day 1 vs day 30 ).

\section{Effect of oral administration of Imm I 24-E on body weight}

Oral administration of Imm124-E led to no change in body weight.

\section{Discussion}

The data presented in our study show that Imm124-E is a safe oral treatment. No side effects were noted in any of the treated patients. Imm124-E led to a clear improvement in 
A

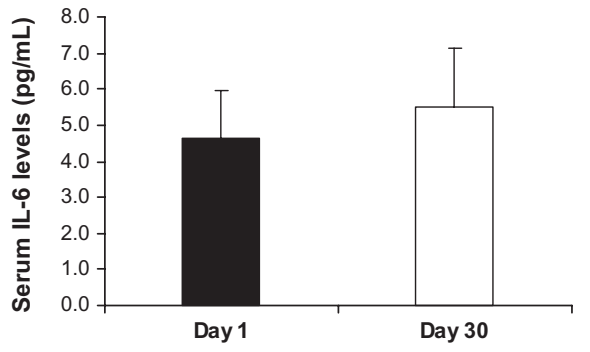

B

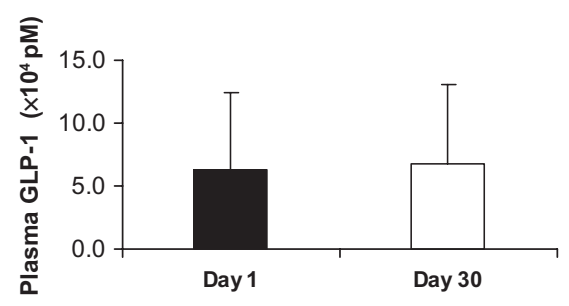

C

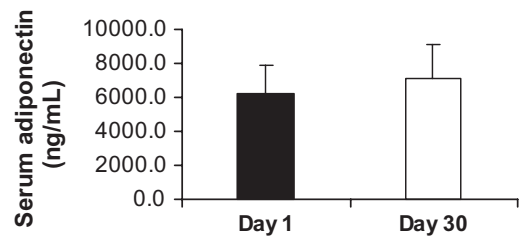

Figure 3 Effect of Imm I24-E on serum levels of IL-6, GLP-I, and adiponectin. All sera were measured using ELISA kits at days I and 30 of the trial in all treated patients. Graphs show (A) Serum levels of IL-6 from six patients; (B) Serum levels of GLP-I post-glucose tolerance test from five patients; (C) Serum levels of adiponectin from eight patients.

Note: Graphs indicate means \pm SD.

Abbreviations: IL-6, interleukin-6; GLP-I, glucagon-like peptide I; ELISA, enzyme-linked immunosorbent assay; SD, standard deviation.

insulin resistance and to alleviation of related liver injury in NASH patients. These effects of Imm124-E were associated with an increased distribution of $\mathrm{T}$ regulatory lymphocytes and an improved lipid profile in treated patients.

Immediately postpartum, high concentrations of various immune components can be found in colostrum, with immunoglobulins making up approximately $5 \%$ of the colostrum content. ${ }^{26}$ Colostrum is also rich in cytokines and other immune agents that provide bacteriostatic, bactericidal, antiviral, anti-inflammatory, and immunoprotection against infection. ${ }^{27,28}$

The value of $\mathrm{BC}$ as a biologic in medicine is well documented. ${ }^{13} \mathrm{BC}$ has been already shown to balance blood sugar levels. The high content of IGF-I in BC, which has been shown to stimulate glucose transport in type 2 diabetes, ${ }^{29}$ may provide a possible explanation for such an effect. An efficient treatment to achieve and sustain glycemic control is measured by improvement in $\mathrm{HbA}_{10}{ }^{30}$ We show a significant decrease of more than $14 \%$ in all ten treated patients, in terms of $\mathrm{HbA}_{\mathrm{lc}}$ values. Reduced fasting glucose levels, and improved HOMA score and OGTT strengthened the enhancement of glycemic control exerted by Imm124-E. Furthermore, oral administration of Imm124-E induced an increase in the levels of circulating GLP-1, a gut hormone that plays an important role in regulating glucose homeostasis by both pancreatic and extrapancreatic mechanisms (defects of GLP-1 in type 2 diabetes result in inappropriately low insulin secretion after oral ingestion of nutrients). ${ }^{25}$

Passive immunization based on bovine colostral preparations is an area of active research. BC-based immune milk products have proven efficacy in prophylaxis and treatment against various infectious diseases in humans,

\section{A}

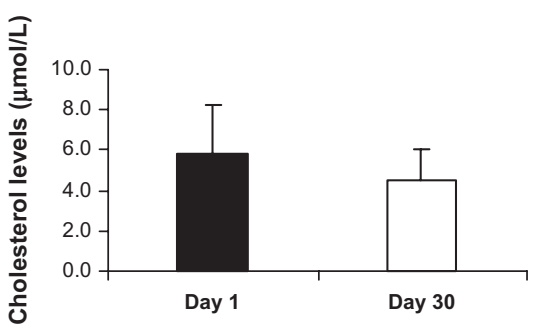

B

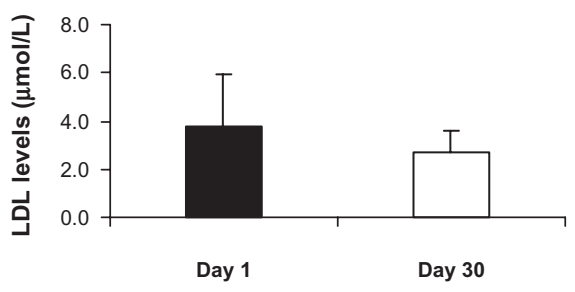

Figure 4 Effect of Imm I24-E on lipid profile. All sera were measured at days I and 30 of the trial in all treated patients. Graphs show (A) Total cholesterol levels from five patients; (B) LDL levels from seven patients.

Note: Graphs indicate means \pm SD.

Abbreviations: LDL, low-density lipoprotein; SD, standard deviation. 
A

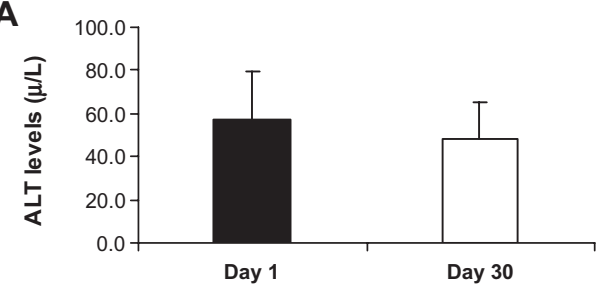

C

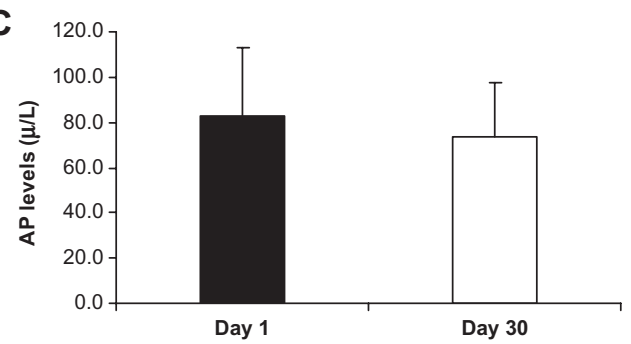

B

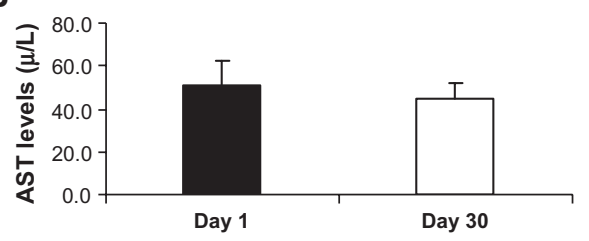

D

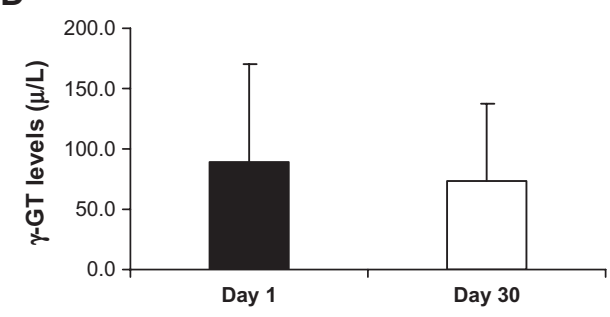

Figure 5 Effect of Imm I24-E on liver enzymes. The serum liver enzymes were measured in treated patients at days I and 30 of the study. Graphs show (A) ALT levels from five patients; (B) AST levels from five patients; (C) AP levels from eight patients; (D) $\gamma$-GT levels from five patients.

Note: Graphs indicate means \pm SD.

Abbreviations: ALT, alanine aminotransferase; SD, standard deviation; AST, aspartate aminotransferase; AP, alkaline phosphatase; $\gamma$-GT, gamma-glutamyl transpeptidase.

such as diarrheal diseases caused by E. coli, rotavirus, and other pathogens. ${ }^{31,32}$ However, the treatment of NASH with colostrum enriched with anti-LPS antibodies has not been previously published. BC contains immunoglobulins that provide the newborn calf with protection against microbial infections until its own immune system matures. The concentration of antibodies against pathogens in colostrum can be raised by immunizing cows with pathogens or their antigens. While normal $\mathrm{BC}$ contains active IgG against specific enteric pathogens, their specificity is dictated by previous systemic challenge, and often the concentration is too low to afford optimal protection..$^{33}$ Therefore, treatment with hyperimmune colostrum is more effective.

LPS and endogenous gut-derived bacterial endotoxins have been suggested to play a role in NASH. The increased permeability in liver disease related to steatohepatitis appears to be caused by the disruption of intracellular tight junctions in the intestine. This has been shown to correlate with a high prevalence of small intestine bacterial overgrowth. ${ }^{34}$ It has also been shown that a lifestyle that included high-intensity and high-volume exercise induced favorable changes in inflammatory markers in relation to endotoxin treatment. ${ }^{35}$ Another study showed that probiotic bacteria prevented hepatic damage and maintained colonic barrier function in a mouse model of sepsis; in this report, orally administered probiotics prevented liver and intestinal damage through a peroxisome proliferatoractivated receptor (PPAR)-dependent mechanism. ${ }^{36}$ In our study, the association between gut-derived bacterial endotoxins and the efficacy of Imm124-E in treating NASH was not established and has to be further studied.
The link between inflammatory and metabolic signaling is a delicate balance. An important feature of inflammation is the infiltration of inflamed tissues by immune cells. ${ }^{37} \mathrm{In}$ the last few years several reports have described the involvement of regulatory $\mathrm{T}$ cells in the cross junction of inflammation, liver disease, and insulin resistance. ${ }^{20,21,24}$ For example, $\mathrm{CD} 4+\mathrm{T}$ lymphocytes resident in the visceral adipose tissue were shown to control insulin resistance in mice with dietinduced obesity. ${ }^{20} \mathrm{Also}$, treatment of humans with anti CD3 was shown to affect Tregs along with alteration in cytokine secretion and PBMC proliferation. ${ }^{38}$ In our study, after one month of oral treatment of Imm124-E, clear changes were found in various subtypes of Tregs. The effect on Tregs was accompanied by an increase in serum IL- 6 and a small increase in adiponectin levels.

A bulk of data has accumulated during the last two decades that supports the notion that insulin resistance in diabetes is primarily a lipid disease. Treatment with Imm124$\mathrm{E}$ had a beneficial effect on the lipid profile and liver injury of treated patients. Also, skim milk from cows immunized with a polyvalent human gut bacterial vaccine was claimed to reduce significantly elevated blood cholesterol and triglyceride concentrations. ${ }^{19}$

This hyperimmune $\mathrm{BC}$ may serve as an easy and safe method for generating antigen-specific antibodies and as a source of immune adjuvants.

In summary, oral administration of Imm124-E is safe and exerts an immunomodulatory effect in patients with type 2 diabetes, hyperlipidemia, and NASH. The anti-inflammatory effect and the promotion of Tregs are associated with 
alleviation of insulin resistance, hyperlipidemia, and liver damage in these patients. Further trials will aim at studying a larger cohort to elucidate the immunomodulatory effect at the level of adipose tissue.

\section{Disclosure}

Y Ilan is the medical director of Immuron. Victor Wong, Brian Muller, Grant Rawlin are employees of Immuron. This study was supported, in part, by Immuron Ltd. Apart from this, the Authors report no conflicts of interest in this work.

\section{References}

1. Neuschwander-Tetri BA, Clark JM, Bass NM, et al; NASH Clinical Research Network. Clinical, laboratory and histological associations in adults with nonalcoholic fatty liver disease. Hepatology. 2010;52(3): 913-924.

2. Daniel S, Ben-Menachem T, Vasudevan G, Ma CK, Blumenkehl M. Prospective evaluation of unexplained chronic liver transaminase abnormalities in asymptomatic and symptomatic patients. Am J Gastroenterol. 1999;94(10):3010-3014.

3. Clark JM, Brancati FL, Diehl AM. Nonalcoholic fatty liver disease. Gastroenterology. 2002;122(6):1649-1657.

4. Sorbi D, McGill DB, Thistle JL, Therneau TM, Henry J, Lindor KD. An assessment of the role of liver biopsies in asymptomatic patients with chronic liver test abnormalities. Am J Gastroenterol. 2000;95(11):3206-3210.

5. Byron D, Minuk GY. Clinical hepatology: profile of an urban, hospitalbased practice. Hepatology. 1996;24(4):813-815.

6. Neuschwander-Tetri BA, Caldwell SH. Nonalcoholic steatohepatitis: summary of an AASLD Single Topic Conference. Hepatology. 2003; 37(5):1202-1219.

7. Almeda-Valdés P, Cuevas-Ramos D, Aguilar-Salinas CA. Metabolic syndrome and non-alcoholic fatty liver disease. Ann Hepatol. 2009; 8 Suppl 1:S18-S24.

8. Clark JM. The epidemiology of nonalcoholic fatty liver disease in adults. J Clin Gastroenterol. 2006;40 Suppl 1:S5-S10.

9. Sanyal AJ, Campbell-Sargent C, Mirshahi F, et al. Nonalcoholic steatohepatitis: association of insulin resistance and mitochondrial abnormalities. Gastroenterology. 2001;120(5):1183-1192.

10. Marchesini G, Brizi M, Bianchi G, et al. Nonalcoholic fatty liver disease: a feature of the metabolic syndrome. Diabetes. 2001;50(8): 1844-1850.

11. Mero A, Miikkulainen H, Riski J, Pakkanen R, Aalto J, Takala T. Effects of bovine colostrum supplementation on serum IGF-I, IgG, hormone, and saliva IgA during training. $J$ Appl Physiol. 1997;83(4):1144-1151.

12. Séverin S, Wenshui X. Milk biologically active components as nutraceuticals: review. Crit Rev Food Sci Nutr. 2005;45(7-8):645-656.

13. Struff WG, Sprotte G. Bovine colostrum as a biologic in clinical medicine: a review - Part II: clinical studies. Int J Clin Pharmacol Ther. 2008;46(5):211-225.

14. Struff WG, Sprotte G. Bovine colostrum as a biologic in clinical medicine: a review. Part I: biotechnological standards, pharmacodynamic and pharmacokinetic characteristics and principles of treatment Int J Clin Pharmacol Ther. 2007;45(4):193-202.

15. Loimaranta V, Tenovuo J, Virtanen S, et al. Generation of bovine immune colostrum against Streptococcus mutans and Streptococcus sobrinus and its effect on glucose uptake and extracellular polysaccharide formation by mutans streptococci. Vaccine. 1997;15(11):1261-1268.

16. Loimaranta V, Nuutila J, Marnila P, Tenovuo J, Korhonen H, Lilius EM. Colostral proteins from cows immunised with Streptococcus mutans/S. sobrinus support the phagocytosis and killing of mutans streptococci by human leucocytes. J Med Microbiol. 1999;48(10):917-926.
17. Howard AN, Marks J. Hypocholesterolaemic effect of milk. Lancet. 1977;2(8031):255-256.

18. Malinow MR, McLaughlin P. The effect of skim milk on plasma cholesterol in rats. Experientia. 1975;31(9):1012-1013.

19. Golay A, Ferrara JM, Felber JP, Schneider H. Cholesterol-lowering effect of skim milk from immunized cows in hypercholesterolemic patients. Am J Clin Nutr. 1990;52(6):1014-1019.

20. Winer S, Chan Y, Paltser G, et al. Normalization of obesityassociated insulin resistance through immunotherapy. Nat Med. 2009;15(8):921-929.

21. Feuerer M, Herrero L, Cipolletta D, et al. Lean, but not obese, fat is enriched for a unique population of regulatory $\mathrm{T}$ cells that affect metabolic parameters. Nat Med. 2009;15(8):930-939.

22. Adar T, Ben Ya' acov A, Lalazar G, et al. Oral administration of immunoglobulin G-enhanced colostrum alleviates insulin resistance and liver injury and is associated with alterations in natural killer T cells. Clin Exp Immunol. 2012;167(2):252-260.

23. DeFronzo RA, Stonehouse AH, Han J, Wintle ME. Relationship of baseline $\mathrm{HbA}_{1 \mathrm{c}}$ and efficacy of current glucose-lowering therapies: a meta-analysis of randomized clinical trials. Diabet Med. 2010;27(3): 309-317.

24. Ilan Y, Maron R, Tukpah AM, et al. Induction of regulatory T cells decreases adipose inflammation and alleviates insulin resistance in ob/ ob mice. Proc Natl Acad Sci U S A. 2010;107(21):9765-9770.

25. Kjems LL, Holst JJ, Vølund A, Madsbad S. The influence of GLP-1 on glucose-stimulated insulin secretion: effects on beta-cell sensitivity in type 2 and nondiabetic subjects. Diabetes. 2003;52(2):380-386.

26. Neville MC. Anatomy and physiology of lactation. Pediatr Clin North Am. 2001;48(1):13-34.

27. Buescher ES, McWilliams-Koeppen P. Soluble tumor necrosis factor-alpha (TNF-alpha) receptors in human colostrum and milk bind to TNF-alpha and neutralize TNF-alpha bioactivity. Pediatr Res. 1998;44(1):37-42.

28. Garofalo RP, Goldman AS. Cytokines, chemokines, and colonystimulating factors in human milk: the 1997 update. Biol Neonate. 1998;74(2):134-142.

29. Dohm GL, Elton CW, Raju MS, et al. IGF-I - stimulated glucose transport in human skeletal muscle and IGF-I resistance in obesity and NIDDM. Diabetes. 1990;39(9):1028-1032.

30. Nathan DM, Buse JB, Davidson MB, et al; American Diabetes Association; European Association for Study of Diabetes. Medical management of hyperglycemia in type 2 diabetes: a consensus algorithm for the initiation and adjustment of therapy: a consensus statement of the American Diabetes Association and the European Association for the Study of Diabetes. Diabetes Care. 2009;32(1):193-203.

31. Korhonen H, Marnila P, Gill HS. Milk immunoglobulins and complement factors. Br J Nutr. 2000;84 Suppl 1:S75-S80.

32. Hammarström L, Weiner CK. Targeted antibodies in dairy-based products. Adv Exp Med Biol. 2008;606:321-343.

33. Hilpert H, Brüssow H, Mietens C, Sidoti J, Lerner L, Werchau H. Use of bovine milk concentrate containing antibody to rotavirus to treat rotavirus gastroenteritis in infants. $J$ Infect Dis. 1987;156(1):158-166.

34. Miele L, Valenza V, La Torre G, et al. Increased intestinal permeability and tight junction alterations in nonalcoholic fatty liver disease. Hepatology. 2009;49(6):1877-1887.

35. Lira FS, Rosa JC, Pimentel GD, et al. Endotoxin levels correlate positively with a sedentary lifestyle and negatively with highly trained subjects. Lipids Health Dis. 2010;9:82.

36. Ewaschuk J, Endersby R, Thiel D, et al. Probiotic bacteria prevent hepatic damage and maintain colonic barrier function in a mouse model of sepsis. Hepatology. 2007;46(3):841-850.

37. Hotamisligil GS. Inflammation and metabolic disorders. Nature 2006;444(7121):860-867.

38. Ilan Y, Zigmond E, Lalazar G, et al. Oral administration of OKT3 monoclonal antibody to human subjects induces a dose-dependent immunologic effect in $\mathrm{T}$ cells and dendritic cells. J Clin Immunol. 2010;30(1):167-177. 
Journal of Inflammation Research

Dovepress

\section{Publish your work in this journal}

The Journal of Inflammation Research is an international, peer-reviewed open-access journal that welcomes laboratory and clinical findings on the molecular basis, cell biology and pharmacology of inflammation including original research, reviews, symposium reports, hypothesis formation and commentaries on: acute/chronic inflammation; mediators of inflamma-

tion; cellular processes; molecular mechanisms; pharmacology and novel anti-inflammatory drugs; clinical conditions involving inflammation. The manuscript management system is completely online and includes a very quick and fair peer-review system. Visit http://www.dovepress.com/ testimonials.php to read real quotes from published authors.

Submit your manuscript here: http://www.dovepress.com/journal-of-inflammation-research-journal 